\title{
Technology-Independent Analysis of the Double Current-Gain Peak in Millimeter-Wave FETs
}

\author{
Giovanni Crupi, Senior Member, IEEE, Antonio Raffo, Member, IEEE, Valeria Vadalà, Member, \\ IEEE, Gustavo Avolio, Member, IEEE, Dominique M. M.-P. Schreurs, Fellow, IEEE, \\ Giorgio Vannini, Member, IEEE, and Alina Caddemi, Member, IEEE
}

\begin{abstract}
This letter is aimed at discovering and analyzing anomalous phenomena affecting millimeter-wave FETs, focusing on a GaN HEMT as a case study. For the first time, we show that the real parts of the impedance parameters can increase and then decrease with frequency, due to the resonance of the extrinsic reactive elements. This resonance may be detected as a peak in the magnitude of the short-circuit current-gain. Such a peak is found to be substantially bias and temperature insensitive and to manifest at frequencies higher than the other current-gain peak, due to the resonance between intrinsic capacitances and extrinsic inductances, giving origin to the double current-gain peak.
\end{abstract}

Index Terms-Current-gain peak, equivalent circuit, scattering parameters, semiconductor device modeling, temperature.

\section{INTRODUCTION}

$\mathrm{U}$ nderstanding anomalies affecting the millimeter-wave FET performance is essential in helping technologists to manufacture devices having the most nearly ideal characteristics, modelers to mimic the real device behavior, and designers to get the targeted circuit specifications. A few relevant examples of these anomalous phenomena are: the kink in the output reflection coefficient $\left(S_{22}\right)$ [1]-[3], the dips in the magnitudes of the impedance (Z-) parameters turning into kinks in the magnitudes of the hybrid $(h-)$ parameters [3]-[6], and the high-frequency increase of the real parts of the Zparameters [7]-[9]. The kink in $S_{22}$ has been mainly ascribed to the relatively high transconductance $\left(g_{m}\right)$ [1]-[3]. The dips in the Z-parameter magnitudes have been ascribed to the resonance between intrinsic capacitances and extrinsic

Manuscript received ; revised

accepted _. This work was supported in part by FWO Vlaanderen, Belgium and Hercules and also performed in the context of the Eurostars project E!10149 MicromodGaN.

Giovanni Crupi is with the Department of Biomedical and Dental Sciences and Morphofunctional Imaging, University of Messina, 98125 Messina, Italy (email: crupig@unime.it).

Antonio Raffo, Valeria Vadalà, and Giorgio Vannini are with the Department of Engineering, University of Ferrara, 44122 Ferrara, Italy.

Gustavo Avolio and Dominique M. M.-P. Schreurs are with the Electronic Engineering Department, KU Leuven, B-3001 Leuven, Belgium.

Alina Caddemi is with the Department of Engineering, University of Messina, 98166 Messina, Italy. inductances [3]-[5]. In particular, the dip in the magnitude of the open circuit output impedance $\left(Z_{22}\right)$ can lead to a peak in the magnitude of the short-circuit current-gain $\left(h_{21}\right)$, which has been referred to as the current-gain peak (CGP) [4]. The highfrequency increase of $\operatorname{Re}\left(Z_{i j}\right)$ has been called PDRZ effect, since it implies a positive derivative of $\operatorname{Re}\left(Z_{i j}\right)$ versus frequency, and its origin has been associated with the concurrence of extrinsic capacitive and inductive effects [7][9]. With the aim of exploring so far uninvestigated anomalies in the millimeter-wave FET performance, this study is focused on the behavior of a $0.25 \times 1500 \mu \mathrm{m}^{2}$ GaN HEMT up to 65 GHz. The choice of investigating a large device at high frequencies is motivated by the fact that the intrinsic terminals tend to be quicker shorted by increasing the frequency and the gate width, thereby enhancing anomalies arising from the extrinsic reactive elements. We will show theoretically and experimentally that the resonance between the extrinsic capacitances and inductances can cause peaks in $\operatorname{Re}\left(Z_{i j}\right)$, due to the transition from PDRZ to NDRZ effect. By contrast to PDRZ, the acronym NDRZ is coined here to refer to the negative derivative of $\operatorname{Re}\left(Z_{i j}\right)$ versus frequency. The peaks in $\operatorname{Re}\left(Z_{i j}\right)$ can turn into peaks in the $Z$-parameter magnitudes. Among these peaks, the one occurring in the magnitude of the open-circuit forward transfer impedance $\left(Z_{2 l}\right)$ can be responsible for a further peak in the magnitude of $h_{21}$ at frequencies higher than the resonance frequency between intrinsic capacitances and extrinsic inductances. Generally, the resonance of the extrinsic reactive elements manifests at a higher frequency, due to the smaller values of the extrinsic capacitances compared to the intrinsic ones. These considerations allow explaining that $h_{21}$ can be affected by the phenomenon called by us double current-gain peak (DCGP). The first CGP depends slightly on temperature and strongly on bias point [3]-[6], whereas the second CGP is almost insensitive to both conditions. It is worth noting that the developed theoretical analysis is technology independent. As a matter of fact, the DCGP is simply due to the circuit element values of the tested FET, besides to the working frequency. This is corroborated by previous studies focused on GaAs and GaN devices affected by the first CGP [3]-[9]. Furthermore, although the second CGP has not so far been observed experimentally, we were able to detect this effect by simulating 
the extracted models beyond the upper frequency limit of our experiments [3]-[5], [7]-[9].

\section{THEORETICAL AND EXPERIMENTAL STUDY OF THE DCGP}

Typically, the small-signal FET equivalent-circuit consists of eight extrinsic elements (i.e., $C_{p g}, C_{p d}, L_{g}, L_{s}, L_{d}, R_{g}, R_{s}$, and $R_{d}$ ) and eight intrinsic elements (i.e., $C_{g s}, C_{g d}, C_{d s}, R_{g s}, R_{g d}, R_{d s}$, $g_{m}$, and $\tau_{m}$ ), as illustrated in Fig. 1. The intrinsic terminals can be assumed to be short-circuited for large devices at high frequencies, due to the reduction of the impedances associated with the intrinsic resistances and capacitances. This assumption enables defining $\operatorname{Re}\left(Z_{11}\right)$ and $\operatorname{Re}\left(Z_{22}\right)$ as follows [7]:

$$
\begin{aligned}
& \operatorname{Re}\left(Z_{11}\right)=\frac{R_{g}+R_{s}}{\left(1-\omega^{2} C_{p g} L_{s g}\right)^{2}} \\
& \operatorname{Re}\left(Z_{22}\right)=\frac{R_{d}+R_{s}}{\left(1-\omega^{2} C_{p d} L_{s d}\right)^{2}}
\end{aligned}
$$

where $L_{s g}$ and $L_{s d}$ are given by $L_{s}+L_{g}$ and $L_{s}+L_{d}$, respectively.

Equations (1) and (2) indicate that the resonance frequencies associated to the peaks in $\operatorname{Re}\left(Z_{11}\right)$ and $\operatorname{Re}\left(Z_{22}\right)$ are:

$$
\begin{aligned}
& f_{R Z 11}=\frac{1}{2 \pi \sqrt{C_{p g} L_{s g}}} \\
& f_{R Z 22}=\frac{1}{2 \pi \sqrt{C_{p d} L_{s d}}} .
\end{aligned}
$$

Along the same lines, similar considerations can be made for $\operatorname{Re}\left(Z_{12}\right)$ and $\operatorname{Re}\left(Z_{21}\right)$ but, since each of these two parameters can be influenced by both $C_{p g}$ and $C_{p d}$, the corresponding equations result to be much less intuitive. According to the aforementioned analysis, the resonance of the extrinsic reactive elements can lead to the transition from a positive to a negative derivative of $\operatorname{Re}\left(Z_{i j}\right)$ versus frequency, implying peaks in the real parts and magnitudes of the $Z$-parameters. It is worth highlighting that the frequency dependence of $Z_{21}$ and $Z_{22}$ can strongly impact on $h_{21}$ (i.e., $h_{21 d B}=Z_{21 d B}-Z_{22 d B}$ ). This observation allows explaining that the magnitude of $h_{21}$ can be affected by the DCGP consisting of two peaks: the first one due to a dip in $Z_{22}$, originating from the resonance between intrinsic capacitances and extrinsic inductances [4], and the second one due to a peak in $Z_{21}$, originating from the resonance between extrinsic capacitances and inductances. The origin of the first CGP has been already demonstrated to be caused by the resonance between $L_{s d}$ and $C_{T}$, given by $C_{d s}$ in parallel with the series capacitance of $C_{g s}$ and $C_{g d}$ [4].

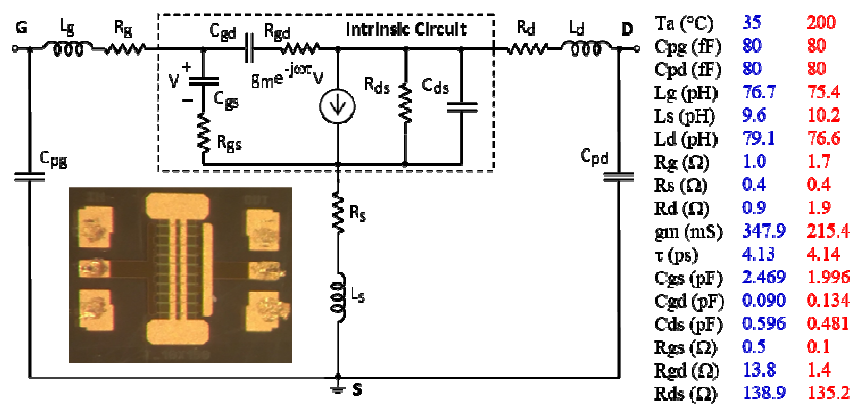

Fig. 1. Standard FET equivalent circuit and element values for the illustrated GaN HEMT at $V_{G S}=-3.1 \mathrm{~V}$ and $V_{D S}=30 \mathrm{~V}$ with $T_{a}=35^{\circ} \mathrm{C}$ and $T_{a}=200^{\circ} \mathrm{C}$.

The present study is based on $S$-parameters measured from 0.2 to $65 \mathrm{GHz}$ on a $0.25 \times 1500 \mu \mathrm{m}^{2} \mathrm{GaN}$ HEMT on $\mathrm{SiC}$ at four ambient temperatures $\left(T_{a}\right): 35^{\circ} \mathrm{C}, 90^{\circ} \mathrm{C}, 145^{\circ} \mathrm{C}$, and $200^{\circ} \mathrm{C}$. An LRM off-wafer calibration is performed using a GGB Industries CS-5 substrate. The extrinsic elements are extracted from $S$-parameters at "cold" pinch-off condition, thereby enabling the determination of the intrinsic elements from the intrinsic admittance ( $Y$-) parameters. The effects of $C_{p g}$ and $C_{p d}$ are de-embedded from the measurements by using a circuit simulator and their values are determined by gradually increasing them from zero until the PDRZ and NDRZ effects disappear from the de-embedded data. The PDRZ and NDRZ effects are found to vanish for all the analyzed $T_{a}$ by deembedding the same extrinsic capacitance values. This is illustrated in Fig. 2 showing the results under "cold" pinch-off condition at the lowest and highest $T_{a}$. The achieved result agrees with the fact that the PDRZ and NDRZ effects origin from the counterbalance between extrinsic inductive and capacitive effects and that the extrinsic capacitances are mostly insensitive to $T_{a}$ [9], [10]. The validity of our theoretical analysis is also confirmed by the fact that the transitions from PDRZ to NDRZ effect in $Z_{11}$ and $Z_{22}$ occur at frequencies around $60 \mathrm{GHz}$ as predicted by (3) and (4) (i.e., $f_{R Z 11}=60.6$ $\mathrm{GHz}$ and $f_{R Z 22}=59.7 \mathrm{GHz}$ at $35^{\circ} \mathrm{C}$ ). The extracted model is able to mimic the measured $S$-parameters and thus to capture the behavior of $h_{21}$ at $V_{G S}=-3.1 \mathrm{~V}$ and $V_{D S}=30 \mathrm{~V}$ (see Fig. 3). As predicted, the resonance of the extrinsic reactive elements leads to a peak in $h_{21}$ at around $60 \mathrm{GHz}$. In particular, the experimental results show peaks in the real parts and magnitudes of all the four $Z$-parameters but the peak in $Z_{21}$ is much more pronounced than the peak in $Z_{22}$, thereby turning into a peak in $h_{21}$ at around $60 \mathrm{GHz}$ (see Fig. 4). Furthermore, a dip in $Z_{22}$ leads to the appearance of the first peak in $h_{21}$. The estimated value of the resonance frequency associated to $L_{s d}$ and $C_{T}$ is $20.4 \mathrm{GHz}$, consistently with the measurements and simulations showing the first CGP at $15.4 \mathrm{GHz}$ and $20.6 \mathrm{GHz}$, respectively. Fig. 4(d) shows that the second CGP occurs at a frequency greater than the unity current-gain cut-off frequency $\left(f_{T}\right)$ and the maximum oscillation frequency $\left(f_{M A X}\right)$ (i.e., $f_{T}=$ $21.2 \mathrm{GHz}$ and $f_{M A X}=36 \mathrm{GHz}$ ). Fig. 5 shows the temperature dependence of the magnitude of $h_{21}$ at four bias conditions. For the sake of completeness, the device is also biased by imposing $I_{D S}$ rather than $V_{G S}$, in order to compensate for 
variations in $I_{D S}$ with $T_{a}$. As can be observed, the second CGP is roughly independent of $T_{a}$ for all the studied bias points, consistently with the fact that the extrinsic capacitances and inductances are mostly insensitive to $T_{a}$ [9], [10]. It is obtained that also the first CGP is roughly independent of $T_{a}$, coherently with the fact that the intrinsic capacitances depend only slightly on $T_{a}$. The first CGP disappears at zero $V_{D S}$, owing to the reduction of $R_{d s}$ that tends to short circuit the intrinsic capacitive effects on $Z_{22}$ [4]. On the other hand, the second CGP is not affected by the reduction of $R_{d s}$, as the resonance of the extrinsic reactive elements occurs at such high frequencies that the intrinsic terminals can be already assumed shorted.

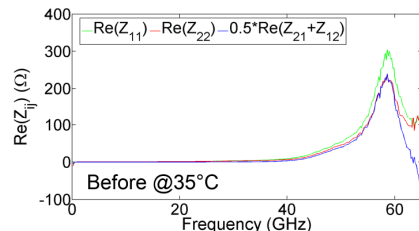

(a)

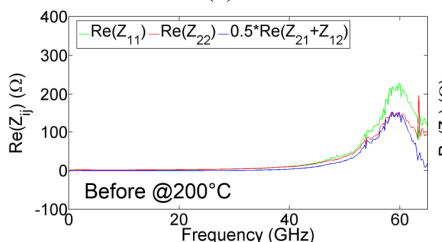

(c)

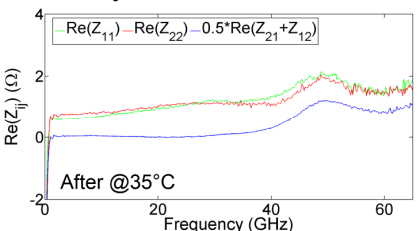

(b)

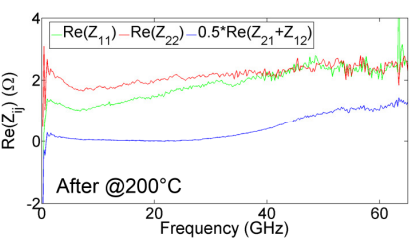

(d)
Fig. 2. Measured $\operatorname{Re}\left(Z_{i j}\right)$ for a GaN HEMT at $V_{G S}=-4 \mathrm{~V}$ and $V_{D S}=0 \mathrm{~V}$ with (a, b) $T_{a}=35^{\circ} \mathrm{C}$ and (c, d) $T_{a}=200^{\circ} \mathrm{C}$ : $(\mathrm{a}, \mathrm{c})$ before and $(\mathrm{b}, \mathrm{d})$ after deembedding the extrinsic capacitances.

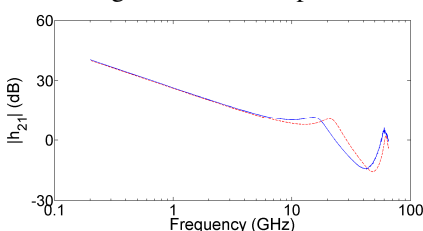

(a)

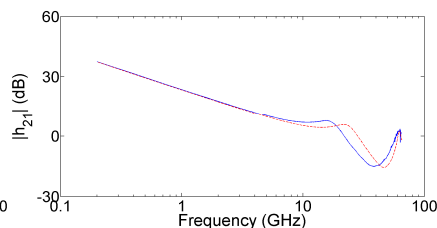

(b)
Fig. 3. Measured (blue lines) and simulated (red lines) $\left|h_{21}\right|$ for a GaN HEMT at $V_{G S}=-3.1 \mathrm{~V}$ and $V_{D S}=30 \mathrm{~V}$ with (a) $T_{a}=35^{\circ} \mathrm{C}$ and (b) $T_{a}=200^{\circ} \mathrm{C}$.

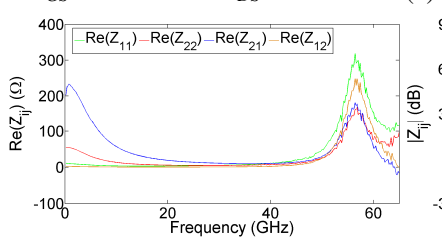

(a)

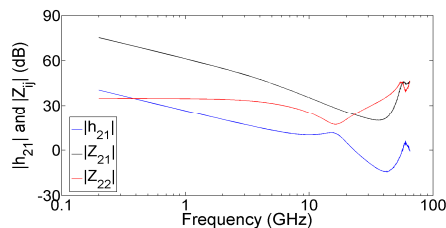

(c)

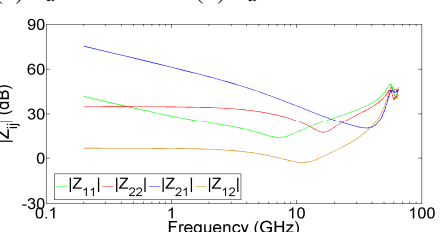

(b)

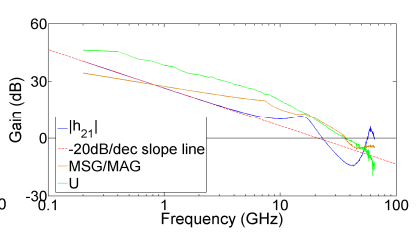

(d)
Fig. 4. Measured (a) $\operatorname{Re}\left(Z_{i j}\right)$, (b) $\left|Z_{i j}\right|$, (c) $\left|h_{21}\right|,\left|Z_{21}\right|$, and $\left|Z_{22}\right|$, (d) $\left|h_{21}\right|$ MSG/MAG, and U for a GaN HEMT at $V_{G S}=-3.1 \mathrm{~V}$ and $V_{D S}=30 \mathrm{~V}$.

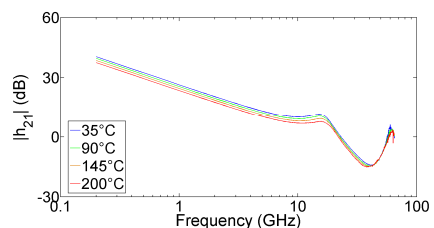

(a)

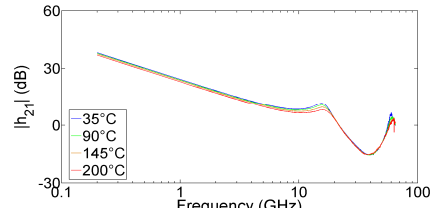

(c)

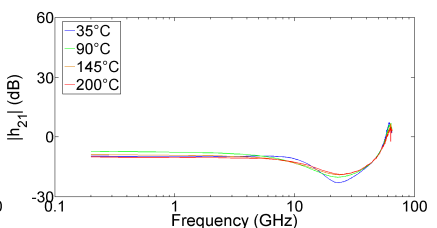

(b)

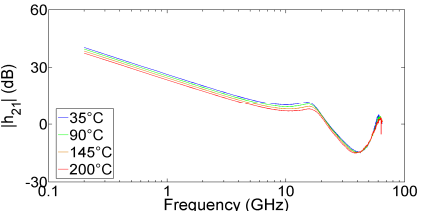

(d)
Fig. 5. Measured $\left|h_{21}\right|$ for a GaN HEMT at four $T_{a}$ and at four bias points: (a) $V_{G S}=-3.1 \mathrm{~V}$ and $V_{D S}=30 \mathrm{~V}$; (b) $V_{G S}=-3.1 \mathrm{~V}$ and $V_{D S}=0 \mathrm{~V}$; (c) $V_{G S}=-3.5$ $\mathrm{V}$ and $V_{D S}=30 \mathrm{~V}$; (d) $I_{D S}=170 \mathrm{~mA}$ and $V_{D S}=30 \mathrm{~V}$.

\section{CONCLUSIONS}

This letter has shown that the studied double current-gain peak phenomenon is inherent in any FET and its onset results in two sudden increases of the current gain at two resonance frequencies, thereby making this effect very attractive. An accurate analysis of the double current-gain peak represents a powerful tool for microwave engineers. For fabrication purpose, electromagnetic simulations of the device layout can properly describe the parasitic reactive network, by which it is possible to predict and control the resonance frequencies before fabricating the device. This enables an optimization of the device layout prior to fabrication, depending on the application constrains. For modeling purpose, the extrinsic capacitances can be determined by exploiting the fact that the PDRZ and NDRZ effects vanish by subtracting appropriate values of extrinsic capacitances. This is an elegant way to overcome the well-known limitation of the conventional "cold" approach that does not allow distinguishing between the extrinsic and intrinsic output capacitances, without the need of using optimization algorithms or additional test structures. For design purpose, the second current-gain peak can enable a current gain greater than unity even at frequencies beyond $f_{T}$ and $f_{M A X}$. This finding extends and complements previous studies in which attention on achieving current-gain peaks even at frequencies beyond $\mathrm{f}_{\mathrm{T}}$ has been restricted to bipolar transistors, mostly based on proper design of the so-called resonance phase transistor.

\section{REFERENCES}

[1] S.-S. Lu, C. Meng, T.-W. Chen, and H.-C. Chen, "The origin of the kink phenomenon of transistor scattering parameter $\mathrm{S}_{22}$," IEEE Trans. Microw. Theory Techn., vol. 49, no. 2, pp. 333-340, Feb. 2001.

[2] S. A. Ahsan, S. Ghosh, S. Khandelwal and Y. S. Chauhan, "Modeling of kink-effect in RF behaviour of GaN HEMTs using ASM-HEMT model," in Proc. of IEEE Int. Conf. on Electron Devices and SolidState Circuits, Hong Kong, China, Aug. 2016, pp. 426-429.

[3] G. Crupi et al., "An extensive experimental analysis of the kink effects in $\mathrm{S}_{22}$ and $\mathrm{h}_{21}$ for a GaN HEMT," IEEE Trans. Microw. Theory Techn., vol. 62, no. 3, pp. 513-520, Mar. 2014.

[4] G. Crupi, A. Raffo, D. Schreurs, G. Avolio, A. Caddemi, and G. Vannini, "A clear-cut understanding of the current-gain peak in 
HEMTs: theory and experiments," Microw. Opt. Tech. Lett., vol. 54, no. 12, pp. 2801-2806, Dec. 2012.

[5] G. Crupi, A. Raffo, D. Schreurs, G. Avolio, A. Caddemi, and G. Vannini, "Identification of the intrinsic capacitive core for GaAs HEMTs by investigating the frequency behavior of the impedance parameters," Microw. Opt. Tech. Lett., vol. 55, no. 6, pp. 2137-1240, Jun. 2013.

[6] S. A. Ahsan, S. Ghosh, S. Khandelwal, and Y. S. Chauhan, "Pole-zero approach to analyze and model the kink in gain-frequency plot of $\mathrm{GaN}$ HEMTs," IEEE Microw. Wireless Comp. Lett., vol. 27, no. 3, pp. 266268, Nov. 2017.

[7] G. Crupi et al., "High-frequency extraction of the extrinsic capacitances for GaN HEMT technology," IEEE Microw. Wireless Comp. Lett., vol. 21, no. 8, pp. 445-447, Aug. 2011.

[8] G. Crupi et al., "In-deep insight into the extrinsic capacitance impact on GaN HEMT modeling at millimeter-wave band," Int. J. RF Microw. Comput. -Aided Eng., vol. 22, no. 3, pp. 308-318, May 2012.

[9] G. Crupi, A. Raffo, G. Avolio, D. Schreurs, G. Vannini, and A. Caddemi, "Temperature influence on GaN HEMT equivalent circuit," IEEE Microw. Wireless Comp. Lett., vol. 26, no. 10, pp. 813-815, Oct. 2016.

[10] M. A. Alim, A. A. Rezazadeh, and C. Gaquiere, "Temperature effect on DC and equivalent circuit parameters of $0.15-\mu \mathrm{m}$ gate length $\mathrm{GaN} / \mathrm{SiC}$ HEMT for microwave applications," IEEE Trans. Microw. Theory Techn., vol. 64, no. 11, pp. 3483-3491, Nov. 2016. 